\title{
Dark Blind Interference Alignment for Downlink of Future Railway Communication Systems
}

\author{
Karine Amis \\ IMT Atlantique \\ UMR CNRS 6285 Lab-STICC, \\ Brest, France \\ karine.amis@imt-atlantique.fr
}

\author{
Thomas Galezowski \\ Société du Grand Paris \\ Paris, France \\ thomas.galezowski@societedugrandparis.fr
}

\author{
Xavier Lagrange \\ IMT Atlantique \\ UMR CNRS 6074 IRISA, \\ Rennes, France \\ xavier.lagrange@imt-atlantique.fr
}

\begin{abstract}
In this paper, we investigate downlink interference management schemes for future railway communications that avoid channel state information (CSI) knowledge at the transmitter side. Assuming reconfigurable antennas at both ends of each train, we apply the dark-blind interference alignment principle. Simulations show that compared to considered orthogonal multiple access techniques, the proposed scheme achieves an efficient trade-off between maximum achievable throughput and error rate.
\end{abstract}

Index Terms-MIMO systems, railway communications, mobile relay, macrodiversity, dark blind interference alignment, reconfigurable antennas

\section{INTRODUCTION}

To face the intensive use of connected devices and to provide high-quality telecommunication services to their passengers, public transports need to adapt and consider the requirement of reliable mobile networks as essential when developing or modifying their infrastructure. Onboard mobile relays is an attractive solution which was investigated and tested [1], [2]. In the perspective of the future Grand Paris Express deployment [3], Société du Grand Paris is interested in developing such technologies [2], [4].

The application of recent cellular standards (long-term evolution (LTE) or even 5G-new radio (5G-NR) [5]) to future railway communication networks defines linear cells, delimited each by two remote radio heads (RRH) that are connected to the same baseband unit pool (BBU). Cooperation between both RRH for transmission and reception is thus possible and macrodiversity [4], [6] is particularly efficient to avoid disastrous degradation of the throughput as the train moves along the section. In this paper, we focus on the downlink and we consider the case when two trains pass each other. Usual multiple-input multiple-output (MIMO) beamforming techniques require channel state information (CSI) knowledge at the transmitter side. The shadowing, involved by one train presence in the neighborhood of the other, could question the CSI accuracy and thus the system performance. To overcome this problem, we investigate downlink interference management schemes, based on RRH cooperation, that avoid CSI knowledge at the transmitter.

This work has been financially supported by Société du Grand Paris.
We also assume that each train is equipped with two distinct reconfigurable antennas, part of two remote radio heads (RRH) connected to the same onboard baseband unit (BBU) thanks to common public radio interface (CPRI) links [7], [8]. Compared to antenna arrays, reconfigurable antennas [9], [10] have the advantages of reduced RF chain number, limited size and lower data volume in transit on the CPRI links, which are of particular interest in our context.

Interference alignment [11] is a non-orthogonal multiple access (NOMA) scheme which achieves asymptotical linear capacity increase with the number of users. Dark-blind interference alignment was introduced in [9] as an alternative scheme to overcome the necessity of global CSI knowledge at any node of the network. It applies to multi-user systems with reconfigurable antenna at the user sides. CSI knowledge is required neither at the transmitter nor at the receiver where simple linear operations enable to cancel multiple-access interference. In this paper, we propose to investigate its use in a railway communication system and compare it to different types of orthogonal multiple access (OMA) schemes.

Our contributions are two-fold. First, we apply dark-blind interference alignment to our context and we propose to distribute it between both transmit RRH. We show that it achieves a good trade-off between maximum achievable throughput and error rate. Second, we investigate the use of reconfigurable antennas with more available modes than required to increase the diversity and thus to improve the robustness of the transmission.

The paper is organized as follows. Section II describes the system model. Section III focuses on the dark-blind interference scheme definition and its theoretical performance. Section IV introduces the OMA schemes, considered for purpose of comparison. Section V is dedicated to simulations and supports the theoretical analysis. Section VI concludes the paper.

\section{A. Notations}

$\boldsymbol{x}$ in bold font and $x$ in normal font stand for a vector and a scalar, respectively. Given an $N \times M$ complex-valued matrix $\boldsymbol{A}$, we denote by $\boldsymbol{A}^{T}, \boldsymbol{A}^{H}, \boldsymbol{A}^{*}$ its transpose, its conjugate transpose and its conjugate, respectively. $\mathbf{0}_{m, n}$ is the zero matrix with $m$ rows and $n$ columns. $\boldsymbol{I}_{m}$ is the identity matrix of size $m$. 


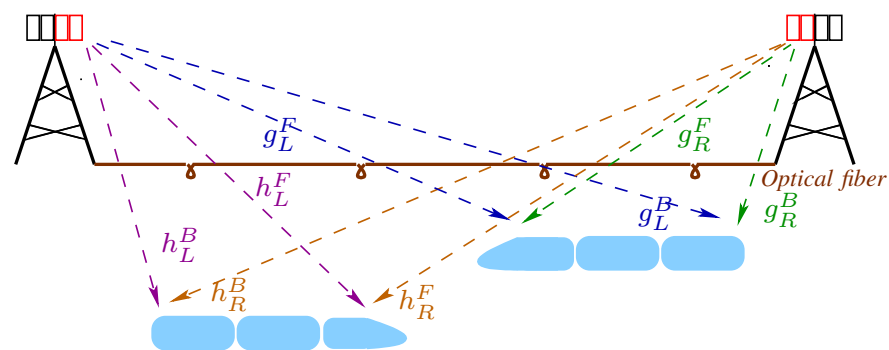

Fig. 1: System model and notations

\section{SYSTEM MODEL}

We consider the downlink of a railway communication system between two trains passing each other on a railway section and two RRH located at each end of the section. One linear cell is defined as the geographical area between two RRHs. We assume that the downlink does not interfere with the uplink (frequency or time division duplexing).

Each section RRH is equipped with two sector antenna arrays (respective coverage area on either side of the RRH). RRHs are assumed to be connected to a remote BBU pool thanks to error-free optical fiber links. Transmit cooperation is thus possible. We denote by $N$ each RRH antenna array element number.

Each train is equipped with two reconfigurable antennas (one on the front, one on the back), part of a RRH. We denote by $M$ the reconfigurable antenna mode number.

We consider a subcarrier index $p$. Without loss of generality and for the sake of simplified notations, we omit $p$ in the remaining of the paper.

The RRH on the left and on the right are referred by the index "L" and "R", respectively. Given one train, we distinguish between the antenna on the front and on the back by "F" and "B", respectively. Let $\boldsymbol{h}_{L}^{F}$ and $\boldsymbol{h}_{R}^{F}$ stand for the 1-by- $N$ subchannel vector between the front of one train and the section RRH on the left and on the right, respectively. Let $\boldsymbol{h}_{L}^{B}, \boldsymbol{h}_{R}^{B}$, stand for the equivalent notations for the back of the train, and $\boldsymbol{g}_{L}^{F}, \boldsymbol{g}_{R}^{F}, \boldsymbol{g}_{L}^{B}, \boldsymbol{g}_{R}^{B}$ for the other train (cf. Fig. 1).

Let us denote by $T_{f}$ the transmission duration. The transmitted frames from both RRH are $N$-by- $T_{f}$ matrices denoted by $\boldsymbol{x}_{L}$ and $\boldsymbol{x}_{R}$, respectively. Then the received frames at the front and at the back of the first train are 1-by- $T_{f}$ vectors denoted by $\boldsymbol{y}_{\boldsymbol{F}}$ and $\boldsymbol{y}_{\boldsymbol{B}}$, respectively.

The transmission can be modeled by

$$
\begin{aligned}
& \boldsymbol{y}_{\boldsymbol{F}}=\boldsymbol{h}_{L}^{F} \boldsymbol{x}_{L}+\boldsymbol{h}_{R}^{F} \boldsymbol{x}_{R}+\boldsymbol{n}_{\boldsymbol{F}}, \\
& \boldsymbol{y}_{\boldsymbol{B}}=\boldsymbol{h}_{L}^{B} \boldsymbol{x}_{L}+\boldsymbol{h}_{R}^{B} \boldsymbol{x}_{R}+\boldsymbol{n}_{\boldsymbol{B}},
\end{aligned}
$$

where $n_{\boldsymbol{F}}$ and $\boldsymbol{n}_{\boldsymbol{B}}$ are length- $T_{f}$ complex-valued noise row vectors. Their components are independent circularlysymmetric Gaussian random variables with zero mean and variance $\sigma^{2}$.

To insist on the RRH cooperation at the transmitter (railway section) and at the receiver side (train), we gather transmitted frames on one hand and, received frames on the other hand.
We introduce $\boldsymbol{x}^{T}=\left[\boldsymbol{x}_{L}^{T} \boldsymbol{x}_{R}^{T}\right], \boldsymbol{y}^{T}=\left[\boldsymbol{y}_{\boldsymbol{F}}^{T} \boldsymbol{y}_{B}^{T}\right]$ and $\boldsymbol{n}^{T}=\left[\boldsymbol{n}_{\boldsymbol{F}}^{T} \boldsymbol{n}_{\boldsymbol{B}}^{T}\right]$. From (1) and (2), we obtain the generic transmission model between both RRH along the railway and the first train:

$$
\boldsymbol{y}=\boldsymbol{H} \boldsymbol{x}+\boldsymbol{n},
$$

where the equivalent channel matrix is given by

$$
\boldsymbol{H}=\left[\begin{array}{ll}
\boldsymbol{h}_{L}^{F} & \boldsymbol{h}_{R}^{F} \\
\boldsymbol{h}_{L}^{B} & \boldsymbol{h}_{R}^{B}
\end{array}\right] .
$$

Similar equations can be derived for the second train.

\section{Distributed DARK-Blind INTERFERENCE ALIGNMENT (DBIA)}

A detailed description of dark-blind interference alignment can be found in [9]. The frame definition is independent of the channel (no necessary CSI knowledge at the transmitter contrary to usual MIMO beamforming techniques). Based on the use of reconfigurable antennas at each user side, this scheme enables to manage the multiple-access interference cancellation thanks to linear combinations of received packets without the need of CSI.

As far as the target application is concerned, the main drawback of such a scheme is the extension of the transmission duration with the user number and/or the antenna modes and the necessity of channel invariance over the frame transmission. Taking this constraint into account, we consider reconfigurable antennas with $M=4$ active modes. According to [9], it enables the transmission of 6 independent packets ( 3 per train) over 15 packet durations. 5G-NR supports different types of numerology [5] and we can adapt the numerology to satisfy the channel variation constraint. In this paper, we adapt the DBIA principle to define a distributed DBIA scheme.

\section{A. Definition}

Let us denote by $\left\{\boldsymbol{p}_{1}, \boldsymbol{p}_{2}, \boldsymbol{p}_{3}\right\}$ and $\left\{\boldsymbol{q}_{1}, \boldsymbol{q}_{2}, \boldsymbol{q}_{3}\right\}$ the packets dedicated to first and second train, respectively. Each packet is a matrix with $2 N$ rows and $T$ columns. In this paper, DBIA is distributed between both RRH along the railway. Each packet is then split into two submatrices according to the rows. For

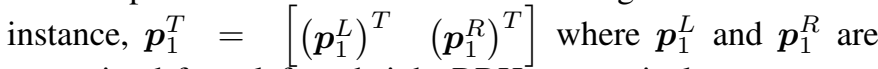
transmitted from left and right RRH, respectively.

Distributed dark-blind interference alignment scheme consists of two successive phases. Orthogonal multiple access is applied in the first phase, while linear combinations of packets are transmitted during the second one. Reconfigurable antennas at the user side change their configuration (minimum period equal to a packet duration) in order to ensure diversity for the user packets and interference cancellation from packets dedicated to the other user.

The left RRH transmits the $N$-by- $T_{f}$ matrix $\boldsymbol{x}_{L}$ defined as $\boldsymbol{x}_{L}=\left[\begin{array}{ll}\boldsymbol{x}_{L}^{1} & \boldsymbol{x}_{L}^{2}\end{array}\right]$ with (see [9] for justification)

$$
\boldsymbol{x}_{L}^{1}=\left[\begin{array}{llllll}
\boldsymbol{p}_{1}^{L} & \boldsymbol{p}_{2}^{L} & \boldsymbol{p}_{3}^{L} & \boldsymbol{q}_{1}^{L} & \boldsymbol{q}_{2}^{L} & \boldsymbol{q}_{3}^{L}
\end{array}\right],
$$


for the first phase and,

$$
\boldsymbol{x}_{L}^{2}=\left[\begin{array}{lllllllll}
\boldsymbol{r}_{11}^{L} & \boldsymbol{r}_{21}^{L} & \boldsymbol{r}_{31}^{L} & \boldsymbol{r}_{12}^{L} & \boldsymbol{r}_{22}^{L} & \boldsymbol{r}_{32}^{L} & \boldsymbol{r}_{13}^{L} & \boldsymbol{r}_{23}^{L} & \boldsymbol{r}_{33}^{L}
\end{array}\right],
$$

for the second phase, where $\boldsymbol{r}_{k j}^{L}=\boldsymbol{p}_{k}^{L}+\boldsymbol{q}_{j}^{L}$.

The right RRH transmits $\boldsymbol{x}_{R}$ similarly. We can observe that each packet is transmitted $M$ times (diversity purpose).

Each reconfigurable antenna has $M$ active modes. Given a train, both antennas apply the same succession of modes (referred to as user signature in the remaining of the paper). For the train whose dedicated packets are $\left\{\boldsymbol{p}_{1}, \boldsymbol{p}_{2}, \boldsymbol{p}_{3}\right\}$, the user signature, denoted by $\mathcal{S}_{1}$, is (see [9] for justification)

$$
\mathcal{S}_{1}=\{4,4,4,1,2,3,1,1,1,2,2,2,3,3,3\} .
$$

We refer to $\boldsymbol{H}(m)$ as the equivalent channel in mode $m$. It means that $\boldsymbol{H} \in\{\boldsymbol{H}(1), \boldsymbol{H}(2), \boldsymbol{H}(3), \boldsymbol{H}(4)\}$ with $\boldsymbol{H}=\boldsymbol{H}(m)$ when mode $m$ is applied. One observe that $\boldsymbol{q}_{j}^{L}$ is always received with mode $j$, which ensures easy interference cancellation. It is achieved by substrating the $(3+j)$-th received length- $T$ packet to each received packet corresponding to the transmission of $\boldsymbol{q}_{j}^{L}$. On the other hand, each dedicated packet $\boldsymbol{p}_{k}^{L}$ is transmitted 4 times and received with a different mode each time (diversity purpose). The second user signature is then defined by

$$
\mathcal{S}_{2}=\{1,2,3,4,4,4,1,2,3,1,2,3,1,2,3\} .
$$

\section{B. Theoretical performance}

We focus on the first train moving from left to right. Given the generic transmission model given in (3), we define $\boldsymbol{y}(t)$ as the submatrix extraction result corresponding to $t$-th packet transmission.

Let us consider the detection of the first packet dedicated to the first train. According to the scheme definition (see (5) and (6)) and given the train signature (see (7)), it requires to process $\boldsymbol{y}(1), \boldsymbol{y}(4), \boldsymbol{y}(5), \boldsymbol{y}(6), \boldsymbol{y}(7), \boldsymbol{y}(10)$ and $\boldsymbol{y}(13)$ :

$$
\begin{aligned}
\boldsymbol{y}(1) & =\boldsymbol{H}(4) \boldsymbol{p}_{1}+\boldsymbol{n}(1), \\
\boldsymbol{y}(4) & =\boldsymbol{H}(1) \boldsymbol{q}_{1}+\boldsymbol{n}(4), \\
\boldsymbol{y}(5) & =\boldsymbol{H}(2) \boldsymbol{q}_{2}+\boldsymbol{n}(5), \\
\boldsymbol{y}(6) & =\boldsymbol{H}(3) \boldsymbol{q}_{3}+\boldsymbol{n}(6), \\
\boldsymbol{y}(7) & =\boldsymbol{H}(1)\left(\boldsymbol{p}_{1}+\boldsymbol{q}_{1}\right)+\boldsymbol{n}(7), \\
\boldsymbol{y}(10) & =\boldsymbol{H}(2)\left(\boldsymbol{p}_{1}+\boldsymbol{q}_{2}\right)+\boldsymbol{n}(10), \\
\boldsymbol{y}(13) & =\boldsymbol{H}(3)\left(\boldsymbol{p}_{1}+\boldsymbol{q}_{3}\right)+\boldsymbol{n}(13) .
\end{aligned}
$$

Linear combinations are used to cancel the interference from packets dedicated to the other train. The detection of the first packet dedicated to the first train is then based on $\boldsymbol{y}(1)$, $\boldsymbol{y}(7)-\boldsymbol{y}(4), \boldsymbol{y}(10)-\boldsymbol{y}(5)$ and $\boldsymbol{y}(13)-\boldsymbol{y}(6)$. Provided perfect CSI knowledge, the four vectors only depend on the packet of interest:

$$
\begin{aligned}
\boldsymbol{y}(1) & =\boldsymbol{H}(4) \boldsymbol{p}_{1}+\boldsymbol{n}(1), \\
\boldsymbol{y}(7)-\boldsymbol{y}(4) & =\boldsymbol{H}(1) \boldsymbol{p}_{1}+(\boldsymbol{n}(7)-\boldsymbol{n}(4)), \\
\boldsymbol{y}(10)-\boldsymbol{y}(5) & =\boldsymbol{H}(2) \boldsymbol{p}_{1}+(\boldsymbol{n}(10)-\boldsymbol{n}(5)), \\
\boldsymbol{y}(13)-\boldsymbol{y}(6) & =\boldsymbol{H}(3) \boldsymbol{p}_{1}+(\boldsymbol{n}(13)-\boldsymbol{n}(6)) .
\end{aligned}
$$

The price to pay is the increase of noise variance on the last three ones (doubled). Similar processing is applied to detect the other two packets, resulting in similar equations.

We assume that the transmitted packets consist of i.i.d. symbols with zero mean and variance $\sigma_{p}^{2}$. Considering (16) to (19), we deduce the maximum achievable throughput per subcarrier and per user, denoted by $C_{D B I A}$ and equal to

$$
C_{D B I A}=\frac{1}{5} \log _{2} \operatorname{det}\left(\boldsymbol{I}_{8}+\frac{\sigma_{p}^{2}}{\sigma^{2}} \boldsymbol{K}^{-1} \widetilde{\boldsymbol{H}} \widetilde{\boldsymbol{H}}^{H}\right),
$$

with

$$
\boldsymbol{K}=\left[\begin{array}{cc}
\boldsymbol{I}_{2} & \mathbf{0}_{2,6} \\
\mathbf{0}_{6,2} & 2 \boldsymbol{I}_{6}
\end{array}\right]
$$

and

$$
\widetilde{\boldsymbol{H}}=\left[\begin{array}{l}
\boldsymbol{H}(4) \\
\boldsymbol{H}(1) \\
\boldsymbol{H}(2) \\
\boldsymbol{H}(3)
\end{array}\right] .
$$

\section{SELECTED REFERENCE SCHEMES}

To investigate the interest of the dark-blind interference scheme, we have selected three other multiple-access schemes that do not require CSI knowledge at the transmitter side and which will be described hereinafter. Extension to generic parameters is straightforward. In all cases, including DBIA, minimum receive diversity is ensured through RRH cooperation on board each train (joint processing of $\boldsymbol{y}_{\boldsymbol{F}}$ and $\boldsymbol{y}_{\boldsymbol{B}}$ ).

\section{A. Orthogonal multiple access with mode selection}

In this scheme, the mode selection means that the best mode (i.e. the best antenna configuration among all available ones) of each reconfigurable antenna is chosen for reception. We refer to this scheme as OMA-T1 (Type 1).

Compared to DBIA, this scheme is limited to the first phase given by (5) with $\boldsymbol{x}_{L} \neq \boldsymbol{x}_{R}$ as for DBIA. First packet dedicated to the first train is thus detected from

$$
\boldsymbol{y}(1)=\boldsymbol{H}(m *) \boldsymbol{p}_{1}+\boldsymbol{n}(1),
$$

where $m^{*}$ means that each reconfigurable antenna applies its best mode configuration.

The maximum achievable throughput is denoted by $C_{O M A-T 1}$ and it is equal to

$$
C_{O M A-T 1}=\frac{1}{2} \log _{2} \operatorname{det}\left(\boldsymbol{I}_{2}+\frac{\sigma_{p}^{2}}{\sigma^{2}} \boldsymbol{H}\left(m^{*}\right) \boldsymbol{H}\left(m^{*}\right)^{H}\right) \text {. }
$$

It achieves better throughput at the expense of the diversity, as each packet is received with only one mode.

\section{B. Orthogonal multiple access with mode diversity}

Compared to DBIA, this scheme, referred to as OMA-T2 (Type 2), repeats $M$ times the first phase given by (5) with $\boldsymbol{x}_{L} \neq \boldsymbol{x}_{R}$ as for DBIA and with a different operating mode at each retransmission. 


\begin{tabular}{|l|l|}
\hline DBIA & Dark blind interference alignment \\
\hline OMA-T1 & $\begin{array}{l}\text { Orthogonal multiple access } \\
\text { with best reconfigurable antenna mode selection }\end{array}$ \\
\hline OMA-T2 & $\begin{array}{l}\text { Orthogonal multiple access } \\
\text { with reconfigurable antenna mode diversity }\end{array}$ \\
\hline OMA-T3 & $\begin{array}{l}\text { Orthogonal multiple access } \\
\text { with best reconfigurable antenna mode selection } \\
\text { and macrodiversity }\end{array}$ \\
\hline
\end{tabular}

TABLE I: Studied schemes

The detection of the first packet dedicated to first train relies on the process of the four following received packets:

$$
\begin{aligned}
\boldsymbol{y}(1) & =\boldsymbol{H}(1) \boldsymbol{p}_{1}+\boldsymbol{n}(1), \\
\boldsymbol{y}(7) & =\boldsymbol{H}(2) \boldsymbol{p}_{1}+\boldsymbol{n}(7), \\
\boldsymbol{y}(13) & =\boldsymbol{H}(3) \boldsymbol{p}_{1}+\boldsymbol{n}(13), \\
\boldsymbol{y}(19) & =\boldsymbol{H}(4) \boldsymbol{p}_{1}+\boldsymbol{n}(19) .
\end{aligned}
$$

It achieves full-diversity (similar to DBIA) at the expense of the throughput (different from OMA-T1). However compared to DBIA, each packet is received without noise variance enhancement, which should yield better error rate performance. The maximum achievable throughput is denoted by $C_{O M A-T 2}$ and it is equal to

$$
C_{O M A-T 2}=\frac{1}{8} \log _{2} \operatorname{det}\left(\boldsymbol{I}_{8}+\frac{\sigma_{p}^{2}}{\sigma^{2}} \widetilde{\boldsymbol{H}} \widetilde{\boldsymbol{H}}^{H}\right) .
$$

C. Orthogonal multiple access with mode selection and macrodiversity

This scheme referred to as OMA-T3 (Type 3) differs from OMA-T1 by the use of macrodiversity for diversity instead of throughput purpose. It means that $\boldsymbol{x}_{L}=\boldsymbol{x}_{R}$ and results in double transmission duration.

The detection of the first packet dedicated to the first train relies on the process of the two following received packets:

$$
\begin{aligned}
& \boldsymbol{y}(1)=\underline{\boldsymbol{H}}\left(m^{*}\right) \boldsymbol{p}_{1}^{L}+\boldsymbol{n}(1), \\
& \boldsymbol{y}(2)=\underline{\boldsymbol{H}}\left(m^{*}\right) \boldsymbol{p}_{1}^{R}+\boldsymbol{n}(2),
\end{aligned}
$$

where $m^{*}$ means that each reconfigurable antenna applies its best mode configuration and the equivalent channel matrix is defined by $\underline{\boldsymbol{H}}=\left[\begin{array}{l}\boldsymbol{h}_{L}^{F}+\boldsymbol{h}_{R}^{F} \\ \boldsymbol{h}_{L}^{B}+\boldsymbol{h}_{R}^{B}\end{array}\right]$.

Denoted by $C_{O M A-T 3}$, the maximum achievable throughput equals

$$
C_{O M A-T 3}=\frac{1}{4} \log _{2} \operatorname{det}\left(\boldsymbol{I}_{2}+\frac{\sigma_{p}^{2}}{\sigma^{2}} \underline{\boldsymbol{H}}\left(m^{*}\right) \underline{\boldsymbol{H}}\left(m^{*}\right)^{H}\right) .
$$

\section{Simulations}

In this section, we compare the selected schemes in terms of maximum achievable throughput and error rate (in that case we consider QPSK modulation). The meaning of acronyms is reported in Table I.

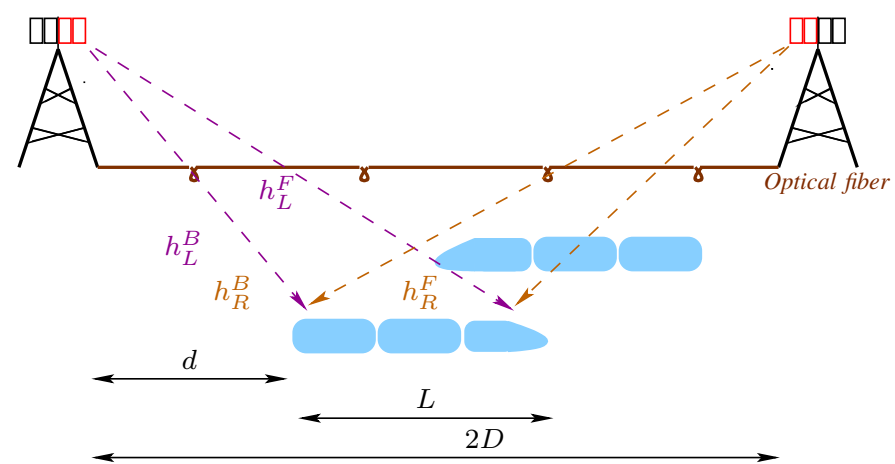

Fig. 2: Notations for simulations

\section{A. Channel model}

Without loss of generality, we focus on the train moving from left to right. The section length is equal to $2 D$ with $D=400$ meters, while the train length $L$ is fixed to 100 meters. $d$ is defined as the distance between left RRH and the back of the train (cf. Fig. 2).

We consider $\boldsymbol{h}_{L}^{B}=\beta \breve{\boldsymbol{h}}_{L}^{B}, \boldsymbol{h}_{L}^{F}=\lambda \breve{\boldsymbol{h}}_{L}^{F}, \boldsymbol{h}_{R}^{B}=\lambda^{\prime} \breve{\boldsymbol{h}}_{R}^{B}$ and $\boldsymbol{h}_{R}^{F}=\beta^{\prime} \breve{\boldsymbol{h}}_{R}^{F}$ where $\breve{\boldsymbol{h}}_{L}^{B}, \breve{\boldsymbol{h}}_{L}^{F}, \breve{\boldsymbol{h}}_{R}^{B}$ and $\breve{\boldsymbol{h}}_{R}^{F}$ are such that their components are i.i.d. complex symmetric Gaussian with zero mean and unitary variance (non-correlated flat Rayleigh fading channel). $\beta, \lambda, \beta^{\prime}$ and $\lambda^{\prime}$ are fixed thanks to the Friis formula in free-space with shadowing parameter $\ell$ such that:

$$
\begin{aligned}
\beta & =\frac{2 D-L}{2 d}, \\
\beta^{\prime} & =\frac{2 D-L}{4 D-2 d-2 L}, \\
\lambda & =\sqrt{\ell} \frac{2 D-L}{2 d+2 L}, \\
\lambda^{\prime} & =\sqrt{\ell} \frac{2 D-L}{4 D-2 d} .
\end{aligned}
$$

The shadowing parameter $\ell$ corresponds to the power loss due to another train coming the other way or due to the other antenna array. When the train moves from left to right, $\beta$ and $\gamma$ decrease while $\beta^{\prime}$ and $\gamma^{\prime}$ increase.

The performance will be studied according to a target signal to noise ratio measured on $y$ (cf. (3)). It would correspond to the OMA scheme applied with arbitrary mode selection (the configuration of the reconfigurable antenna is randomly chosen among all available ones), full shadowing $(\ell \rightarrow 0)$ and the train located in the middle of the section (worst case). To satisfy the resulting constraint on the average channel power, we have also imposed that when the train is located in the middle of the section ( $d=D-\frac{L}{2}$ ), $\beta=\beta^{\prime}=1$ (yielding to the definitions (33)-(36)).

We define the relative position of the train center by $\Delta=$ $\frac{2 d+L}{4 D}$ (The location in the middle of the section corresponds to $\Delta=0.5$ ).

Reconfigurable antennas may have more available configurations than required. To take this feature into account, we can 
select the $M$ best modes among the available ones. As $M$ is small, we can expect $M$ quite uncorrelated modes, meaning better diversity. We investigate the impact of mode diversity on the performance both in terms of throughput and error rate.

Performance in terms of BER for $\Delta=0.5$ and $\Delta=0.25$ are reported in Fig. 3 and in Fig. 4, respectively. As theoretically expected, OMA-T1 and OMA-T3 achieve the same diversity order with a significant coding gain $(6.5 \mathrm{~dB}$ for $\Delta=0.5$ and 9 $\mathrm{dB}$ for $\Delta=0.25)$ for the second one thanks to macrodiversity (transmission of the same packet from both RRH). OMA-T2 and DBIA achieve the same diversity order, higher than the other two, with a loss of less than $3 \mathrm{~dB}$ for DBIA due to noise enhancement involved by interference cancellation. The gain achieved by DBIA over OMA-T1 is significant: measured to roughly $11 \mathrm{~dB}(\Delta=0.25) / 15.5 \mathrm{~dB}(\Delta=0.5)$ for a BER of $10^{-5}$, it gets higher as the BER decreases.

In Fig. 5, we compare OMA-T3 and DBIA for $\Delta=0.25$ and different parameters. We observe that OMA-T3 outperforms DBIA below a given threshold, beyond which the situation is reversed. DBIA takes better profit from increased available mode number (4 selected among 4 or 16). For instance, for a shadowing of $3 \mathrm{~dB}$, the increase of available modes enables a gain of $4 \mathrm{~dB}$ against $2 \mathrm{~dB}$ for OMA-T3. We also measure the impact of shadowing. Let us mention that a shadowing of $100 \mathrm{~dB}$ means $\boldsymbol{h}_{L}^{F}=\boldsymbol{h}_{R}^{B}=\mathbf{0}_{1, N}$, that is to say a reduced receive diversity. For $\Delta=0.25$, it implies that $\boldsymbol{x}_{R}$ is received with a very poor signal power in the case of DBIA, while repetition in OMA-T3 ensures some robustness towards shadowing.

Performance in terms of maximum achievable throughput in bits per channel use (pcu) per subcarrier per user is plotted in Fig. 6 for $\Delta=0.5$, and in in Fig. 7 for $\Delta=0.25$, both with a shadowing parameter of $3 \mathrm{~dB}$ and 4 available modes. We observe that OMA-T2 and OMA-T3 perform roughly the same (the difference decreases with $\Delta$ ) while OMA-T1 outperforms the other three. DBIA performance is in-between. For example, for $\Delta=0.5$ and a target SNR of $20 \mathrm{~dB}$, it achieves a gain of 2 bits compared to OMA-T3 and a loss of 2 bits compared to OMA-T1.

In Fig. 8, we compare OMA-T1 and DBIA for $\Delta=0.25$ and different available mode numbers. We observe that DBIA takes slightly better benefit from an increased available mode number to improve the thoughput, above all when going from 4 to 16 available modes.

In brief, DBIA enables a good tradeoff between data rate (OMA-T1 > DBIA > OMA-T3) and transmission reliability (OMA-T1 $<<$ DBIA in all cases and OMA-T3 $<<$ DBIA beyond an SNR threshold whose value depends on the configuration). Simulations, reported in this paper and carried out in a theoretical framework, enable to draw behaviour trends, which should be refined thanks to experiments in real conditions. In practice, a careful design of reconfigurable antennas would be necessary to ensure mode diversity (number and variety).

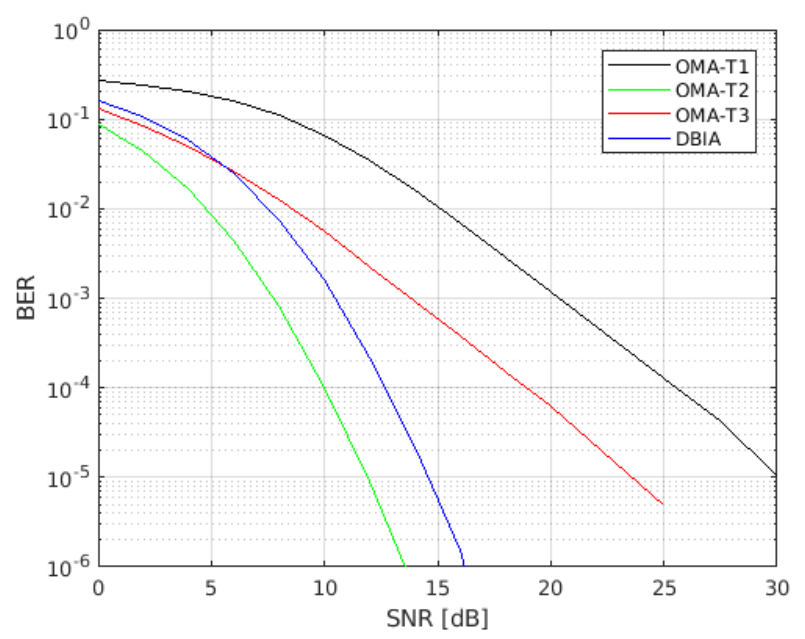

Fig. 3: Comparison in terms of BER $-\Delta=0.5$ - Shadowing of $3 \mathrm{~dB}-4$ modes (see SNR definition in V-A)

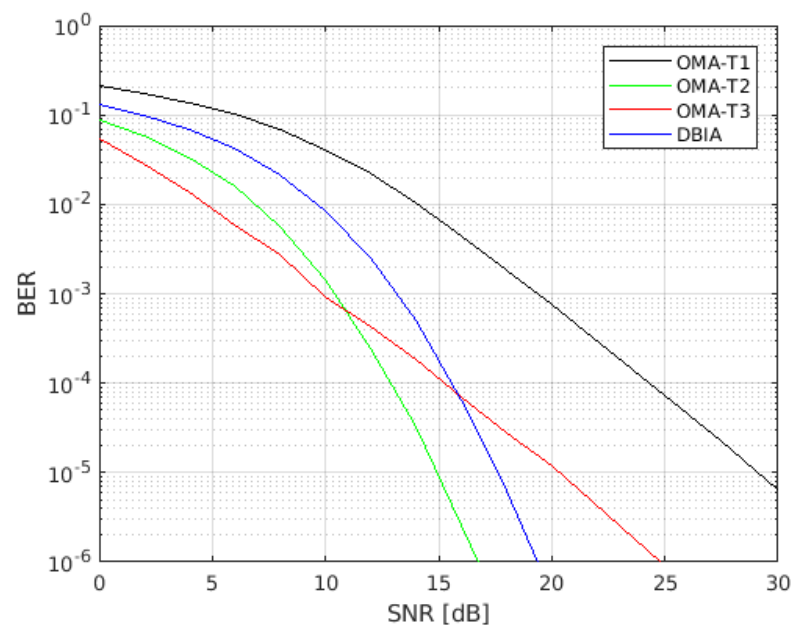

Fig. 4: Comparison in terms of BER - $\Delta=0.25$ - Shadowing of $3 \mathrm{~dB}-4$ modes (see SNR definition in V-A)

\section{CONCLUSION}

In this paper, we considered railway communications with mobile relays onboard and we focused on the downlink towards embedded eNodeB. We assumed that trains are equipped with reconfigurable antennas and that the RRH along the railway cooperate. We investigated multiple-access techniques that do not require CSI knowledge at the transmitter. We showed that distributed dark-blind interference alignment scheme with reconfigurable antenna mode diversity (more available antenna configurations than required) achieves a good trade-off between throughput, error rate and performance stability as the train moves. Future work will investigate the scheme robustness towards channel variation.

\section{REFERENCES}

[1] R. Chen, W-X. Long, G. Mao and C. Li, "Development Trends of 


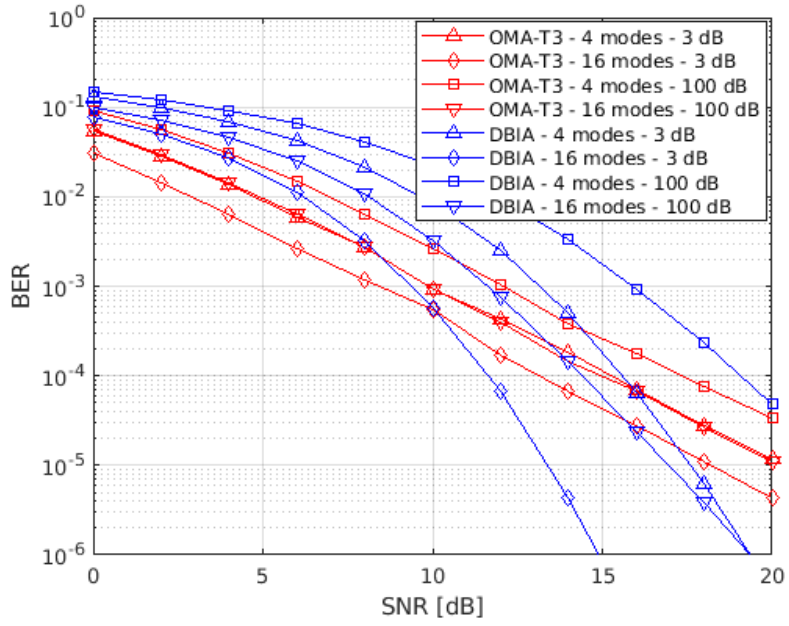

Fig. 5: Comparison in terms of BER $-\Delta=0.25$ - Impact of mode diversity and shadowing (see SNR definition in V-A)

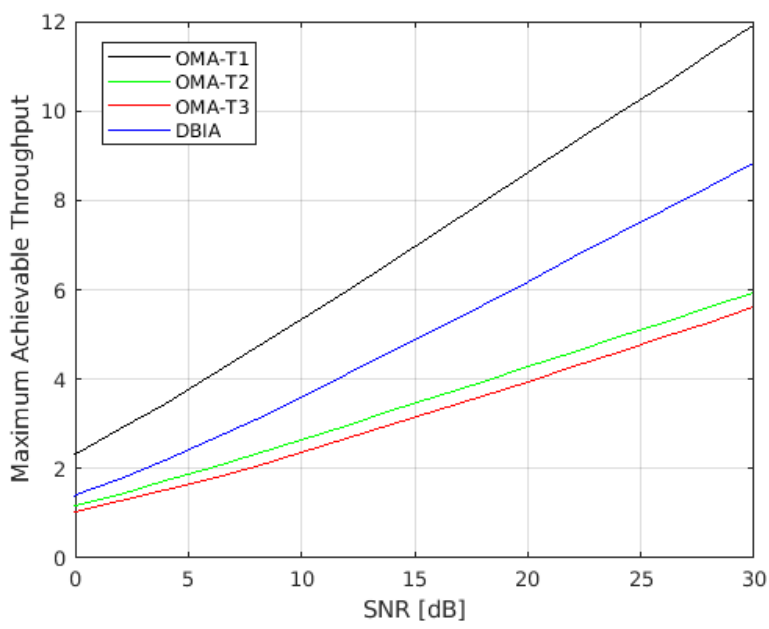

Fig. 6: Comparison in terms of maximum achievable throughput per subcarrier per user [unit: bits pcu] $-\Delta=0.5-$ Shadowing of $3 \mathrm{~dB}-4$ modes (see SNR definition in V-A)

Mobile Communication Systems for railways", IEEE Commun. Surveys and Tutorials, vol. 20, pp.3131-3141, Jul. 2018

[2] T. Kerdoncuff, T. Galezowski and X. Lagrange, "Mobile relay for LTE: proof of concept and performance measurements", IEEE 87th Veh. Technol. Conf., July 2018

[3] Société du Grand Paris, île de France mobilités and Grand Paris express, "Matériels roulants du Grand Paris Express : Le marché entre dans son ultime phase", Press release, May 2018

[4] K. Amis, T. Galezowski and X. Lagrange, "MIMO Cooperation Schemes for Uplink of Future Railway Communication Systems", IEEE 31st Annual Int. Symp. on Personal, Indoor and Mobile Radio Commun., August 2020

[5] F. Hasegawa, A. Taira, G. Noh, B. Hui, H. Nishimoto, A. Okazaki, A. Okamura, J. Lee, and I. Kim, "High-Speed Train Communications Standardization in 3GPP 5G NR", IEEE Commun. Standards Mag., pp.44-52, Mar. 2018

[6] I. Rivas, L.J. Ibbetson and L.B. Lopes, Macrodiversity reception performance investigation in microcellular networks, IEEE 47th Veh. Technol. Conf. Technology in Motion, May 1997

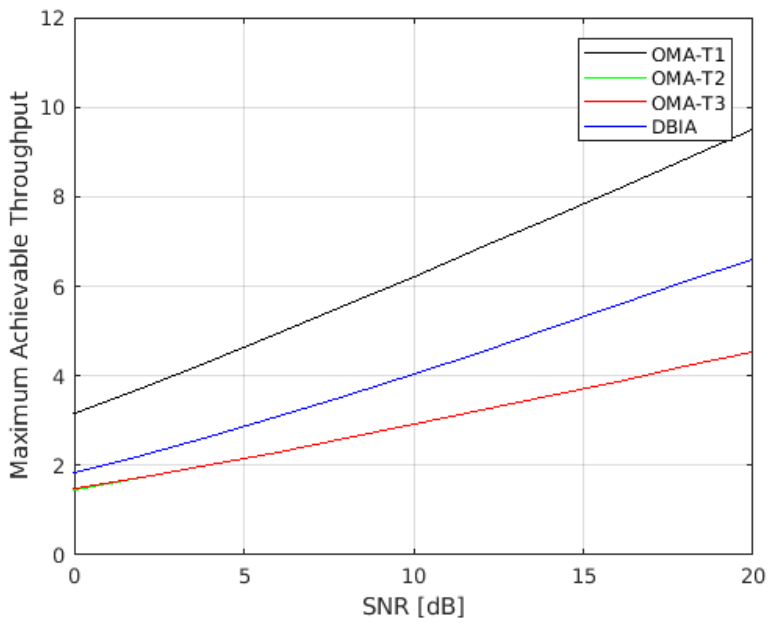

Fig. 7: Comparison in terms of maximum achievable throughput per subcarrier per user [unit: bits pcu] - $\Delta=0.25$ Shadowing of $3 \mathrm{~dB}-4$ modes (see SNR definition in V-A)

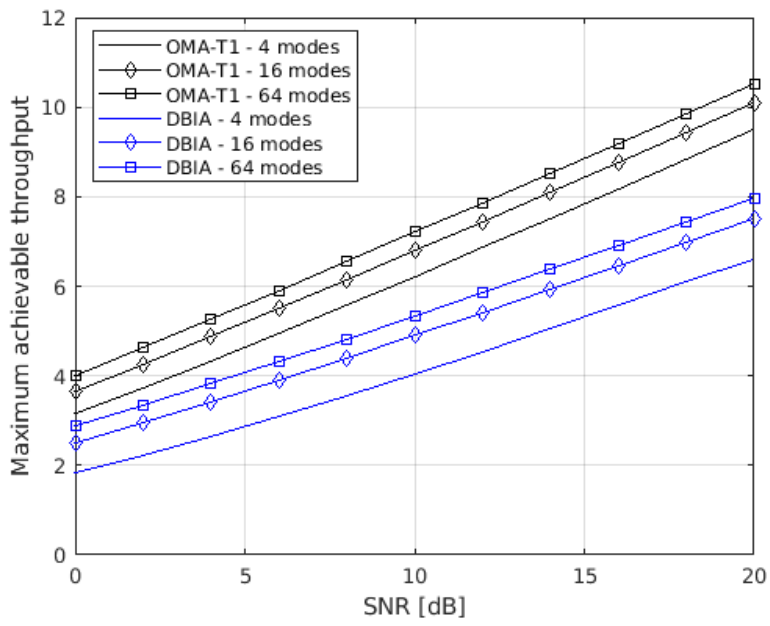

Fig. 8: Comparison in terms of maximum achievable throughput per subcarrier per user [unit: bits pcu] $-\Delta=0.25$ - Impact of mode diversity (see SNR definition in V-A)

[7] Ericsson, Huawei, NEC, and Nokia, "Common Public Radio Interface (CPRI); Interface Specification, V7.0", http://www.cpri.info/downloads/CPRI_v_7_0_2015-10-09.pdf, Oct. 2015

[8] A. de la Oliva, J.A. Hernandez, D. Larrabeiti and A. Azcorra, "An Overview of the CPRI Specification and its Application to C-RANBased LTE Scenaris", IEEE Commun. Mag., vol. 54, no. 5, pp.152-159, Feb. 2016,

[9] T. Gou, C. Wang and S. A. Jafar, "Aiming perfectly in the Dark-Blind Interference Alignment through Staggered Antenna Switching", IEEE Trans. on Signal Process., vol. 59, no. 6, pp. 2734-2744, Jun. 2011

[10] Y. J. Guo, P-Y Qin, S-L Chen, W. Lin and R W. Ziolkowski, ”Advances in Reconfigurable Antenna Systems Facilitated by Innovative Technologies", IEEE Access, vol. 6, pp.5780-5794, Jan. 2018

[11] V. R. Cadambe and S. A. Jafar, "Interference alignment and degrees of freedom of the K-user interference channel", IEEE Trans. on Inf. Theory, vol. 54, no. 8, pp.3425-3441, Aug. 2008 